\title{
The Novel Selective Pan-TRK Inhibitor ONO-7579 Exhibits Antitumor Efficacy Against Human Gallbladder Cancer In Vitro
}

\author{
MAKOTO KAWAMOTO ${ }^{1,2}$, KEIGO OZONO ${ }^{3}$, YASUHIRO OYAMA ${ }^{1,2}$, \\ AKIO YAMASAKI ${ }^{1,2}$, YOSHINAO ODA ${ }^{3}$ and HIDEYA ONISHI ${ }^{1}$ \\ Departments of ${ }^{1}$ Cancer Therapy and Research, ${ }^{2}$ Surgery and Oncology, and \\ ${ }^{3}$ Anatomic Pathology, Graduate School of Medical Sciences, Kyushu University, Fukuoka, Japan
}

\begin{abstract}
We previously reported that brain-derived neurotrophic factor (BDNF)/neurotrophic receptor tyrosine kinase 2 (NTRK2/TRKB) signaling contributes to induction of malignant phenotype of gallbladder cancer $(G B C)$. Recently, pan-TRK inhibitors have been evaluated and their dramatic clinical activity is being shown for a variety of cancer types harboring an NTRK rearrangement in phase I trials. ONO-7579 is an oral pan-TRK inhibitor currently under investigation in phase I/II clinical trial for TRKrearranged solid tumors. In this study, we evaluated the anticancer effect of ONO-7579 using GBC cells with or without KRAS mutant, NOZ, TYGBK-1. Our study showed that ONO-7579 had a suppressive effect on GBC proliferation in TYGBK-1, and on invasive potential and vascular endothelial growth factor expression in TYGBK-1 and NOZ. Our data indicated that ONO-7579 could be a promising treatment option for patients with $G B C$.
\end{abstract}

Gallbladder cancer (GBC) is a fatal disease with poor prognosis. Complete surgical resection is the only potentially curative treatment; however, most GBC cases have developed into locally advanced disease or have metastasized by the time of diagnosis. In inoperable cases, many patients must rely on chemotherapy and radiation therapy, which are not sufficiently effective, and for which the available regimens are still limited. The 5-year survival of patients with stage 0 , I and II GBC was reported to be $80 \%, 50 \%$ and $28 \%$, respectively (1). However, survival rates were found to

Correspondence to: Hideya Onishi, MD, Ph.D., Department of Cancer Therapy and Research Graduate School of Medical Sciences, Kyushu University, Maidashi 3-1-1, Higashi-ku, Fukuoka 812-8582, Japan. Tel: +81 926426220, Fax: +81 926426221, e-mail: ohnishi@surg1.med.kyushu-u.ac.jp

Key Words: ONO-7579, TRK inhibitor, TRKB, gallbladder cancer. be markedly decrease in patients diagnosed with stage III and IV GBC $(<10 \%)$ (2). Therefore, development of novel molecular therapeutic targets is strongly required for improving outcomes for patients with GBC.

Brain-derived neurotrophic factor (BDNF) and its tyrosine kinase receptor neurotrophic receptor tyrosine kinase 2 (NTRK2/TRKB) have been shown to contribute to aggressive phenotype in a wide range of cancer types, including of the head and neck (3), lung (4), ovary (5), pancreas (6), and colon (7). Our previous study demonstrated its contribution to the malignant phenotype of GBC (8). In this study, we evaluated whether specifically targeting TRK would suppress the malignant phenotype of GBC in vitro.

TRK gene fusion was recently recognized as oncogenic in various solid cancer types, including sarcoma (9), non-small cell lung cancer (10), and colorectal cancer (11). Furthermore, pan-TRK inhibitors have been evaluated and dramatic clinical activity is being shown for a variety of cancers harboring an NTRK rearrangement in phase 1 trials (12). ONO-7579 is oral pan-TRK inhibitor and currently under investigation in phase I/II clinical trial for solid tumors. It is expected to inhibit phosphorylation of all TRKs with a highly efficacy and selectivity. Our previous studies demonstrated BDNF/TRKB signaling contributes to the malignant phenotype of GBC (8). Therefore, we believe the potential efficacy of TRK inhibitor would be good news for patients with GBC, for which available approaches are still limited.

In the present study, we examined the effect of ONO-7579 on GBC malignancy in vitro and demonstrated its ability to inhibit BDNF/TRKB-induced proliferation, invasiveness and vascular endothelial growth factor (VEGF) expression.

\section{Materials and Methods}

Cell lines. We used two GBC cell lines for their evident KRAS status: NOZ (13) and TYGBK-1 (14). NOZ and TYGBK-1 were purchased from the Japanese Collection of Research Bioresources bank (Tokyo, Japan). Both cell lines were cultured according to the 
supplier's specifications. There was no mycoplasma contamination in either of the cell lines with mycoplasma detection kit (Lonza, Basel, Switzerland). Cells were cultured in $5 \% \mathrm{CO}_{2}$ and $95 \%$ air, and for hypoxic conditions, cells were cultured in $1 \% \mathrm{O}_{2}, 5 \% \mathrm{CO}_{2}$, and $94 \% \mathrm{~N}_{2}$, in a multigas incubator (Sanyo, Tokyo, Japan).

Cell proliferation assay. GBC cell lines were seeded onto 96-well plates at 5000 cells/well and incubated with TRK inhibitor ONO7579 (gifted by Ono Pharmaceutical Co., Ltd., Osaka, Japan) at 0 , $100,500,1000,2000$, or $3000 \mathrm{nM}$, protein kinase B (AKT) inhibitor MK2206 (Cayman Chemical, Ann Arbor, MI, USA) at 0, 0.01, 0.1, 0.5 or $1 \mathrm{nM}$, or mitogen-activated protein kinase kinase (MEK) inhibitor PD325901 (Cayman Chemical,) at 0, 0.1, 1, 10, 100, or $1,000 \mathrm{nM}$ for $72 \mathrm{~h}$. Cell proliferation was assessed by Biotrak visible plate reader (Amersham Biosciences, Piscataway NJ, USA) at $492 \mathrm{~nm}$ (reference wavelength $620 \mathrm{~nm}$ ) using Cell Count Reagent SF (Nacalai Tesque, Kyoto, Japan).

Cell invasion assay. The invasiveness of the GBC cell lines was assessed by Matrigel invasion assay as described previously (15). Briefly, cells $\left(2 \times 10^{5}\right)$ were placed in falcon tissue culture inserts $(8$ $\mu \mathrm{m}$ pores; Becton Dickinson, Lincoln Park, NJ) with ONO-7579 at $0,100,500,1,000,2,000$, or $3,000 \mathrm{nM}$ and subsequently incubated for $18 \mathrm{~h}$. The cells that migrated to the lower side of the filter were fixed and stained with Diff-Quik reagent (Sysmex, Kobe, Japan) and then counted under a light microscope (Nikon Eclipse TE 300; Nikon, Tokyo, Japan).

Western blot analysis. Western blotting was performed as described previously (15). Cells were prepared for protein extraction after 24$\mathrm{h}$ incubation with BDNF (Peprotech, Rocky Hill, NJ, USA), ONO7579 , PD325901, or MK2206 alone or in combination. The proteincarrying membranes were then incubated overnight at $4^{\circ} \mathrm{C}$ with primary antibodies for TRKB (sc-8316, 1:200), E-cadherin (sc7870, 1:200), vimentin (sc-6260, 1:200), SLUG (sc-15391, 1:200), SNAIL (sc-10433, 1:200), TWIST (sc-15393,1:200), hypoxiainducible factor $1 \alpha$ (HIF-1 $\alpha)$ (sc-8711, 1:100), VEGF (sc152,1:100), VEGFC (sc-7133,1:200), MEK1/2 (sc-436, 1:200), phospho-MEK1/2 (sc-7995, 1:200), AKT1/2/3 (sc-8312, 1:200), or phospho-AKT1/2/3 (sc-101629, 1:200), from Santa Cruz Biotechnology (Santa Cruz, CA, USA); phospho-extracellular signal-regulated kinase (p-ERK1/2) (no. 9101,1:200) from Cell Signaling Technology (Beverly, MA, USA); or p-TRKB (ab197072, 1:500) from Abcam (Cambridge, MA, USA). Peroxidase-linked secondary antibodies (Amersham Biosciences) were subsequently added and the membranes were further incubated for $1 \mathrm{~h}$ at room temperature. Antibody for $\alpha$-tubulin (1:1,000; Sigma-Aldrich, St. Louis, MO, USA) was used as quantitative control protein.

Immunohistochemistry. Tissue samples were obtained from patients with GBC who underwent resection at the Department of Surgery and Oncology, Kyushu University Hospitals, Fukuoka, Japan between 2001 and 2012. Approval for the use of tissues was obtained from patients in accordance with the Ethical Committees for Clinical Study at Kyushu University (approval number: 29-134). Immunohistochemical staining was performed using 4- $\mu \mathrm{m}$-thick formalin-fixed, paraffinembedded tissue sections and primary antibody for TRKB (sc-8316, 1:50; Santa Cruz Biotechnology). Endogenous peroxidase activity was blocked for 30 minutes using methanol containing $0.3 \%$ hydrogen peroxidase. Sections were incubated with primary antibody overnight at $4^{\circ} \mathrm{C}$, followed by incubation for 40 minutes with the secondary antibody at room temperature. The reaction products were visualized using diaminobenzidine (DAB). All slides were examined by microscopy (Carl Zeiss, Tokyo, Japan).

Statistical analysis. All data are represented as mean \pm standard deviation (SD). Student's $t$-test was used for comparison of mean values between two groups. A value of $p<0.05$ was considered significant.

\section{Results}

Effect of ONO-7579 on the TRKB expression in GBC cell lines. TRKB expression was detected in resected human GBC specimens (Figure 1A) and GBC cell lines TYGBK-1 and NOZ (Figure 1B). Addition of BDNF significantly increased TRKB phosphorylation in GBC cells and the effects were abrogated by ONO-7579. TYGBK-1 is known to have wild-type KRAS and NOZ has KRAS mutation. As expected, addition of rhBDNF induced MEK phosphorylation, and ONO-7579 treatment abrogated this effect in TYGBK-1 cells, whereas in NOZ cells, MEK phosphorylation status was unchanged by BDNF or ONO-7579 treatment (Figure 1C). On the basis of this result, we used these cell lines to assess the effect of ONO-7579 on GBC malignancy in subsequent experiments.

Effect of ONO-7579 on GBC proliferation. To study the effects of ONO-7579 on GBC proliferation, TYGBK-1 and NOZ cells were treated with ONO-7579 and cell viability was evaluated by MTS assay. ONO-7579 treatment showed the tendency to suppress proliferation in a dose-dependent manner in TYGBK-1 cells, but not in NOZ (Figure 2A). To investigate the mechanism of this difference, we examined the role of AKT and ERK pathway, known to be major players downstream of BDNF/TRKB signaling (16). MK2206 (AKT inhibitor) and PD325901 (MEK inhibitor) were used to inhibit AKT and ERK pathway, respectively. Optimal drug concentration gradient was determined as shown in Figure 2B. PD325901 suppressed TYGBK-1 proliferation, on the contrary, PD325901 and MK2206 suppressed the NOZ proliferation, respectively (Figure 2C and D). These results suggest that $\mathrm{NOZ}$ proliferation mainly depends on the ERK pathway, which ONO-7579 does not affect in these cells because of KRAS mutation. Collectively, these results indicated that ONO-7579 may have antiproliferative effect on GBC cells harboring wild-type $K R A S$.

Effect of ONO-7579 on BDNF/TRKB-induced GBC migration and invasion. Our previous study indicated that BDNF-induced activation of TRKB contributes to invasive phenotype in GBC cells (8). To determine whether ONO-7579 altered GBC invasive phenotype, migration and invasion assays were performed on NOZ and TYGBK-1 cells treated with ONO7579. ONO-7579 treatment significantly inhibited the 
A i

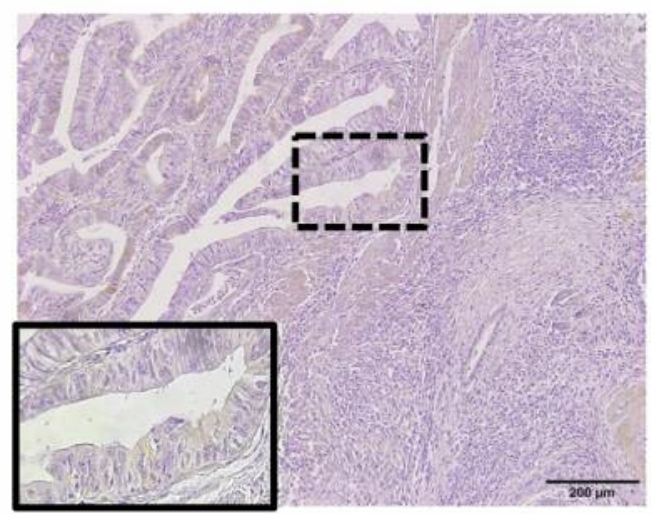

ii

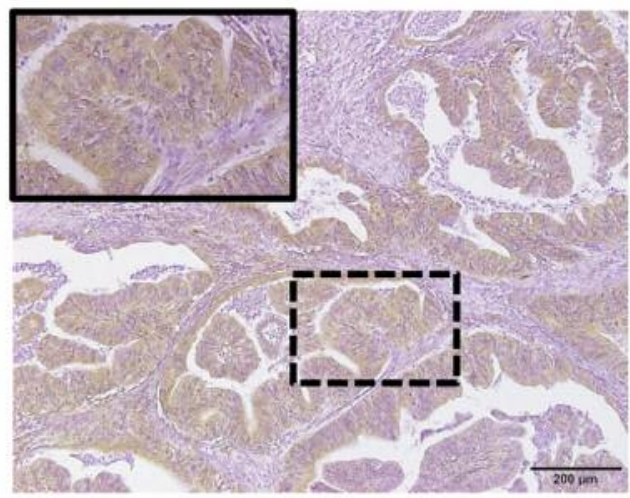

B

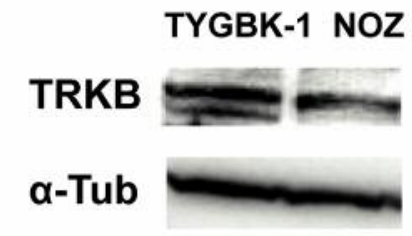

C

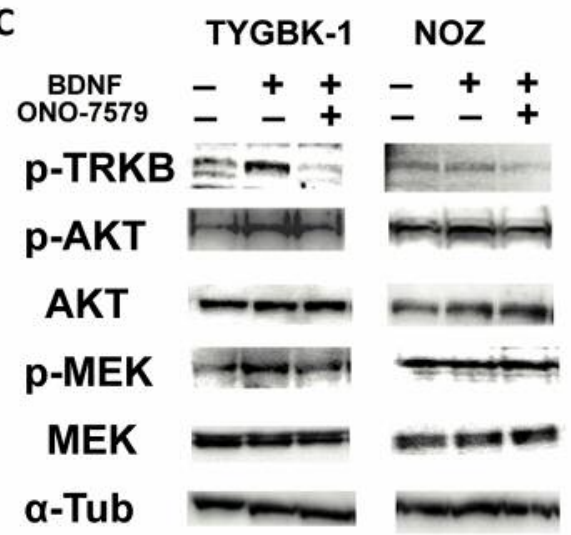

Figure 1. Neurotrophic receptor tyrosine kinase 2 (NTRK2/TRKB) expression in resected human primary gallbladder cancer (GBC) specimens and two GBC cell lines. A: Representative immunohistochemical images of low (i), and high (ii) TRKB expression in GBC specimens. Main image magnification: 100x, inset: 200x. B: Western blot analysis of TRKB in GBC cell lines. C: ONO-7579 abrogated brain-derived neurotrophic factor (BDNF)-induced protein kinase B (AKT) phosphorylation in both GBC cell lines. Treatment with ONO-7579 abrogated BDNF-induced mitogenactivated protein kinase kinase (MEK) phosphorylation in KRAS wild-type TYGBK-1 cells, although addition of recombinant human BDNF or ONO7579 did not affect the MEK phosphorylation status in KRAS-mutant NOZ cells. $\alpha$-Tub: $\alpha$-Tubulin.

migration and invasion of GBC cells in both cell lines (Figure 3 ). This trend is particularly notable for TYGBK-1, cells with wild-type KRAS. These data indicate that ONO-7579 inhibited BDNF/TRKB-induced migration and invasiveness, particularly in GBC cells harboring wild-type $K R A S$.

Effect of ONO-7579 on the expressions of VEGF/VEGFC. Our previous study showed that TRKB expression increases under hypoxic conditions and BDNF/TRKB signaling is involved in VEGF expression via HIF-1 $\alpha$ (17). Based on this, we examined whether ONO-7579 altered the expression of VEGFs. Both cell lines were incubated with ONO-7579 under hypoxic conditions for 24 hours. As shown in Figure 4, ONO-7579 reduced the expression of HIF- $1 \alpha$ and VEGFs in a dose-dependent manner. These data indicate that ONO-7579 inhibited BDNF/TRKBinduced VEGF expression under hypoxic conditions, such to those found in the cancer microenvironment.
Effect of ONO-7579 on BDNF/TRKB-induced epithelialmesenchymal transition (EMT) in GBC cell lines. In our previous study, we reported BDNF/TRKB signaling induces EMT by up-regulating transcription factors (SLUG, SNAIL, and TWIST) in GBC (8). In the present study, we evaluated the effect of ONO-7579 on EMT induction in GBC cells. First we examined the morphological changes of ONO-7579treated cells by analyzing the cell aspect ratio (Figure 5A). In phase-contrast images, we found that control cells showed a spindle-like morphology, which is one of the major characteristics of mesenchymal cells. In contrast, cells treated with ONO-7579 were notably rounded, with epithelial morphology. ONO-7579 treatment substantially reduced the percentage of spindle-shaped cells, and the cells seem to gain cell-cell contacts (Figure 5A).

As shown in Figure 5B, ONO-7579 exposure enhanced Ecadherin expression and reduced vimentin expression in both 

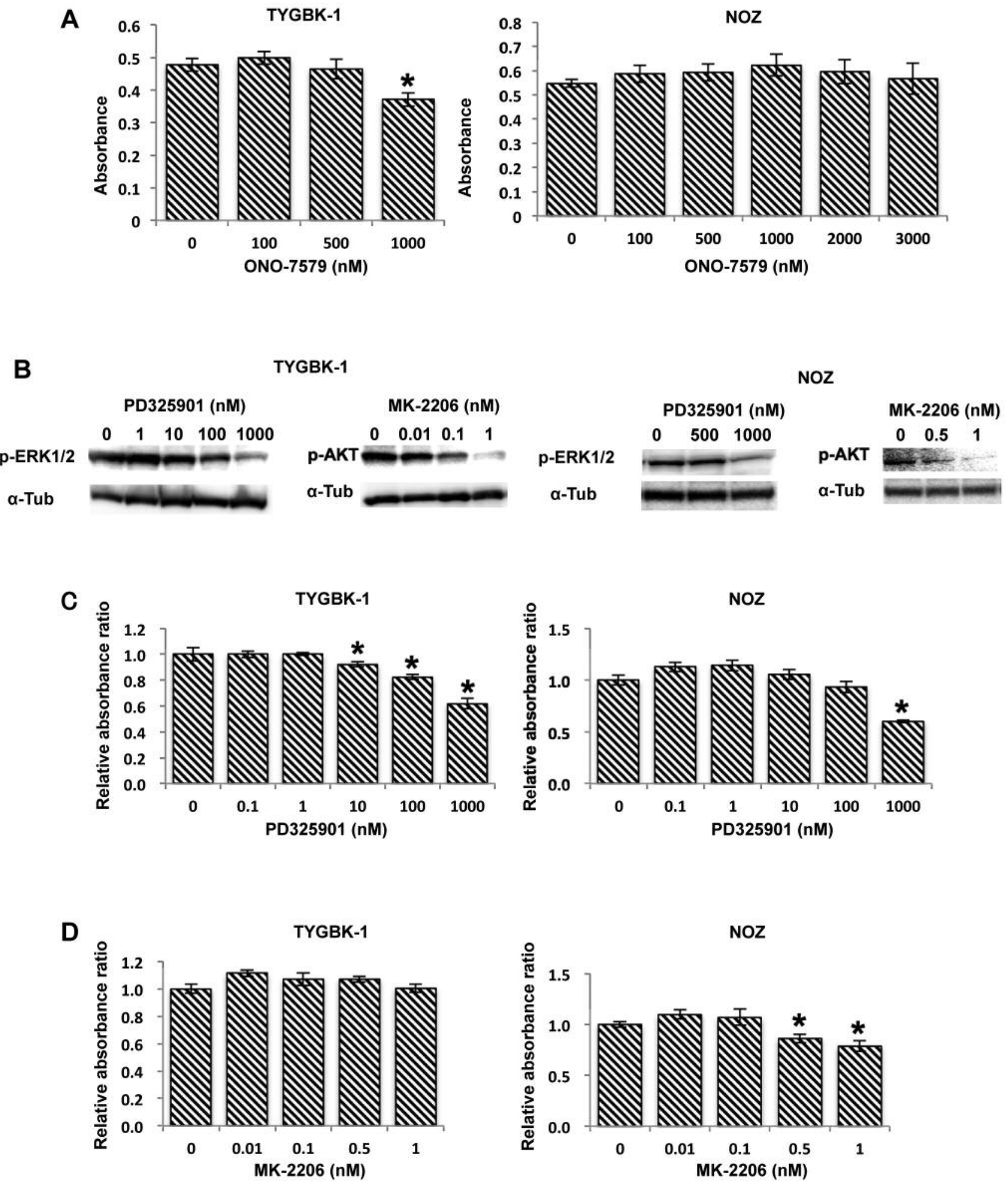

Figure 2. ONO-7579 inhibited the proliferation of gallbladder cancer $(G B C)$ cells. A: Proliferation assay of two GBC cell lines incubated with ONO-7579 for $72 \mathrm{~h}$ at the indicated concentrations. Absorbance correlates with viable cell number, reflecting proliferative ability. B: Optimal drug concentration gradient on treatment with mitogen-activated protein kinase kinase inhibitor PD325901 or protein kinase B (AKT) inhibitor MK-2206. C: Proliferation assay of two GBC cell lines incubated with PD325901 for $72 \mathrm{~h}$ at the indicated concentrations. D: Proliferation assay of two GBC cell lines incubated with MK-2206 for $72 \mathrm{~h}$ at the indicated concentrations. Data are mean $\pm S D$. *Significantly different at $p<0.05$. p-ERK: phospho-extracellular signal-regulated kinase; p-AKT: phospho-protein kinase B; $\alpha$-Tub: $\alpha$-tubulin. 

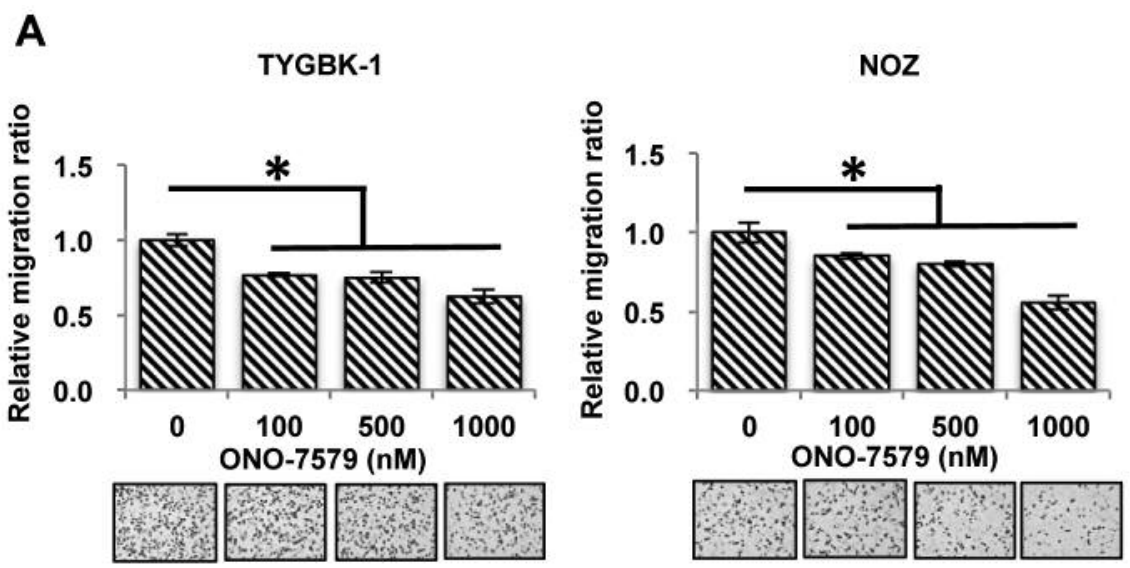

B

TYGBK-1

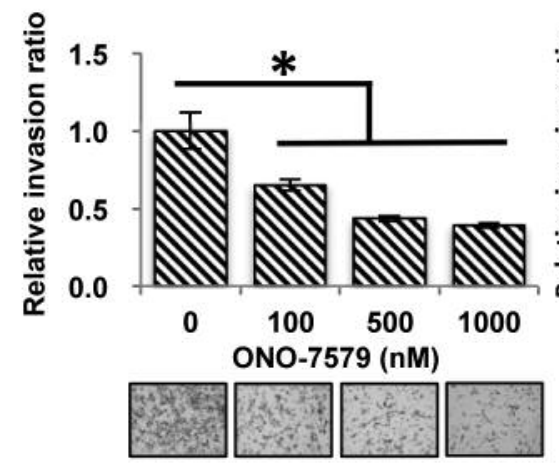

NOZ

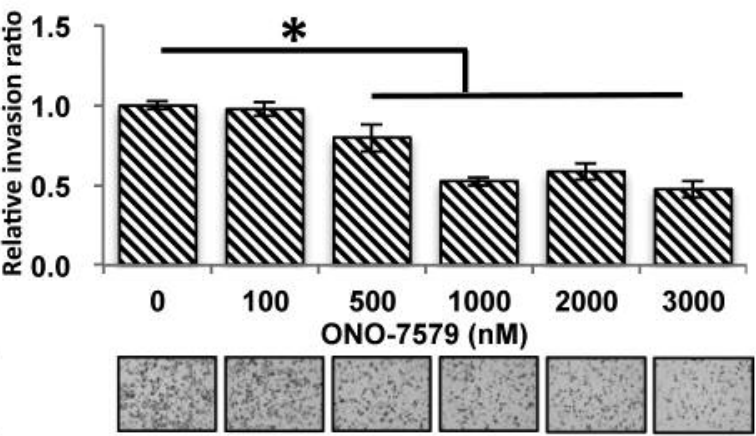

Figure 3. ONO-7579 inhibited migration and invasion in gallbladder cancer (GBC) cells. A: Migration assay of two GBC cell lines incubated for $6 \mathrm{~h}$ with ONO-7579 at the indicated concentrations. B. Invasion assay of two GBC cell lines incubated for 18 hith ONO-7579 at the indicated concentrations. Original magnification: 100x. *Significantly different at $p<0.05$. Data are mean $\pm S D$.

cell lines. Expression of EMT transcription factors (SLUG, SNAIL, and TWIST) also markedly decreased in TYGBK-1 in a dose-dependent manner, but not clearly in NOZ other than SLUG (Figure 5B). Furthermore, we examined the role of AKT and ERK pathway in EMT in GBC cells, that are also known to play critical roles in tumor invasion and metastasis (16). As shown in Figure 5C, TWIST and SNAIL were activated through the AKT pathway and SLUG were ERK pathway in TYGBK-1 cells. On the contrary, these transcription factors appear to be mainly activated through ERK pathway in NOZ cells. This finding could be one of the causes of the difference between TYGBK-1 and NOZ cells in the inhibitory effect on invasiveness. Taken together, these results demonstrate that ONO-7579 suppressed BDNF/TRKBinduced GBC invasiveness via inhibition of EMT by downregulating transcription factors (SLUG, SNAIL, and TWIST), particularly in GBC cells harboring wild-type KRAS.
TYGBK-1

$$
\text { ONO-7579 (nM) } 05001000
$$

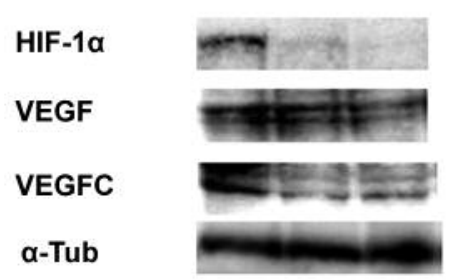

Figure 4. ONO-7579 inhibited the expression of vascular endothelial growth factors (VEGFs) in gallbladder cancer (GBC) cells. Western blot analysis of hypoxia-inducible factor $1 \alpha(H I F-1 \alpha), V E G F$, and VEGFC in two GBC cell lines under hypoxic conditions after 24-h ONO-7579 treatment at the indicated concentrations. $\alpha$-Tub: $\alpha$-Tubulin. 

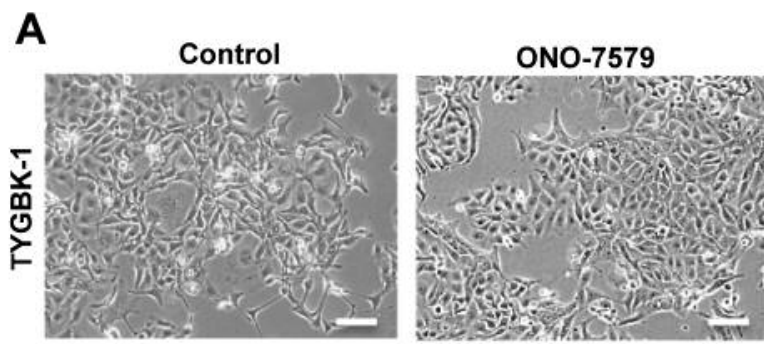

B

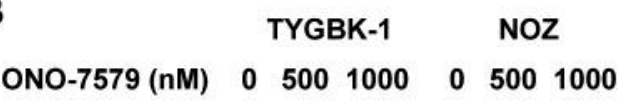
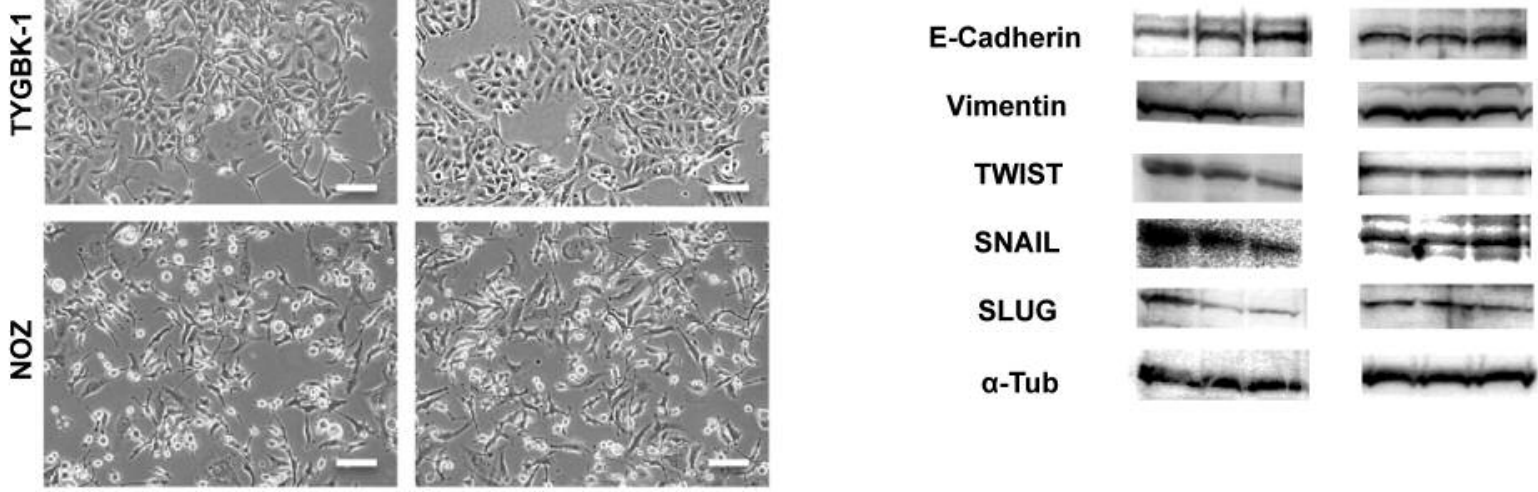

TYGBK-1

NOZ
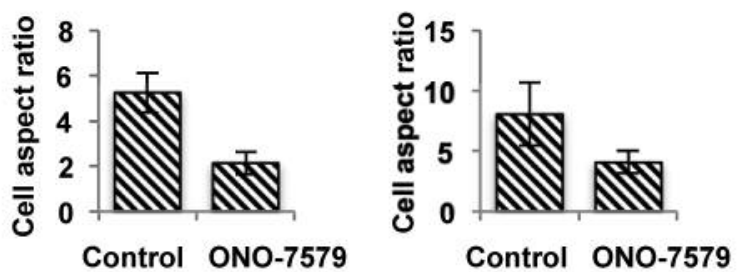

C

TYGBK-1

NOZ

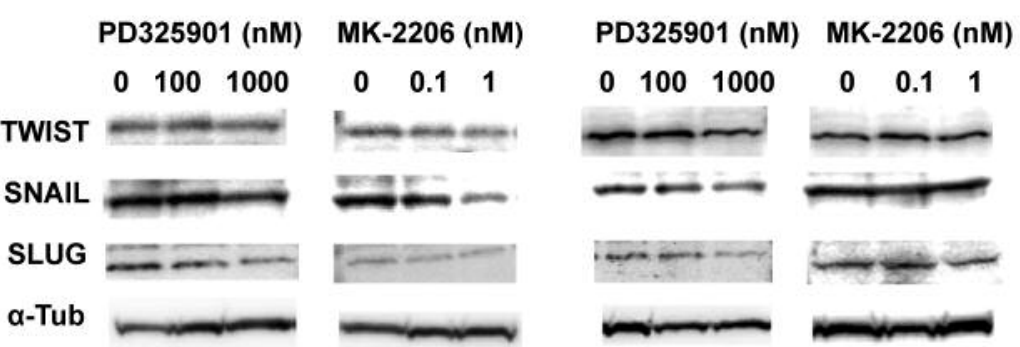

Figure 5. ONO-7579 reduced invasiveness of gallbladder cancer $(G B C)$ cells through inhibiting epithelial-mesenchymal transition (EMT) via the extracellular signal-regulated kinase (ERK) or protein kinase B (AKT) pathway. A: Images showing cell morphological changes after 24-h ONO7579 treatment $(1,000 \mathrm{nM})$ compared to the control (top). Original magnification: 100x. Scale bar: $100 \mu \mathrm{m}$. Bar graph showing cell aspect ratio of the above images (bottom). B: Western blot analysis of E-cadherin, vimentin, and EMT-related transcription factors (SLUG, SNAIL, and TWIST) in two GBC cell lines after 24-h ONO-7579 treatment at the indicated concentrations. C: Western blot analysis of EMT-related transcription factors (SLUG, SNAIL, and TWIST) in two GBC cell lines after 24- $h$ treatment with mitogen-activated protein kinase kinase inhibitor PD325901 or protein kinase B (AKT) inhibitor MK-2206 at indicated concentration. $\alpha$-Tub: $\alpha$-Tubulin.

\section{Discussion}

The TRK family has three isoforms (TRKA, TRKB, and TRKC) that are encoded by NTRK1, NTRK2, and NTRK3, respectively (18). Although the oncological roles of TRKA, TRKB and TRKC have been shown to be different among tumor types despite their close homology, many reports have shown a relationship between TRKB and malignancy of various solid tumors (3-7). Activation of BDNF/TRKB signals induces the activation of down-stream AKT, ERK (13), and HIF- $1 \alpha$ signals (17), which elicits the differential regulation of various cellular activities, such as proliferation (19), differentiation (20), apoptosis (21), and invasion (16). Thus, BDNF/TRKB signaling could be a promising therapeutic target. We previously reported TRKB is potential therapeutic target in some cancer types, including large cell neuroendocrine carcinoma (22), squamous cell carcinoma (23) and GBC (8). 


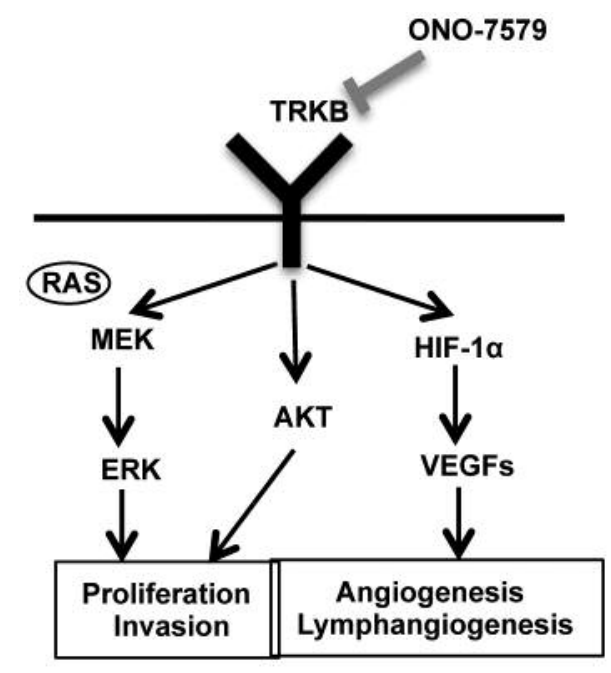

Figure 6. Model of our findings in this study. TRKB: Neurotrophic receptor tyrosine kinase 2 (NTRK2); RAS: rat sarcoma viral oncogene homolog; MEK: mitogen-activated protein kinase kinase; ERK: extracellular signal-regulated kinase; AKT: protein kinase B; HIF-1 $\alpha$ : hypoxia-inducible factor $1 \alpha$; VEGFs: vascular endothelial growth factors.

$\mathrm{K} 252 \mathrm{a}$, which was used in many previous studies as a pan-TRK inhibitor, has shown activity against a range of TRKB-expressing cancer types. However, K252a inhibits several additional targets such as hepatocyte growth factor receptor (MET), protein kinase $\mathrm{C}$ (PKC) and platelet-derived growth factor receptors (PDGFR) (24-25). Therefore, development of potent and selective TRK kinase inhibitors remains critical in order to develop treatment of patients with GBC. Recently, Larotrectinib (LOXO-101), a selective panTRK inhibitor, showed dramatic clinical activity in patients with a variety of cancer types harboring an NTRK rearrangement in phase I trials (12). Furthermore, the acquisition of secondary mutations in the TRK kinase domain after treatment with entrectinib is becoming clear (26). ONO-7579, a second-generation pan-TRK inhibitor, is expected to be highly potent and selective oral-pan-TRK inhibitor with efficacy against disease with resistant mutations NTRK1 G595R and G667C (26). No reports have yet shown the efficacy of ONO-7579 for GBC.

We previously reported that TRKB expression in the invasive front correlates with invasion depth and poor patient survival in GBC (8). As mentioned above, the 5-year survival rates differ greatly between patients with stage II and stage III GBC, at $28 \%$ and $<10 \%$, respectively $(1,2)$. These findings indicate inhibition of invasiveness and lymphatic metastasis are critical for GBC treatment. The potentially suppressive effects on invasion and lymphovascular metastasis convince us that ONO-7579 would be a powerful treatment option, leading to enhanced outcomes for patients with GBC.
$K R A S$ mutation status testing has been highlighted for determination of treatment plan in recent years, and molecular target therapies, such as anti-EGFR therapy, have been developed for several solid cancer types, but not for GBC (27). A previous report showed that KRAS mutations were found in $19-40 \%$ of patients with GBC (28), and a recent report showed that therapies targeting downstream factors of KRAS may be feasible for patients with KRAS mutations (29). Unfortunately, ONO-7579 did not show satisfactory anticancer effect against KRAS-mutant NOZ cells in this experiment.

A potential limitation of our experiment is that we were unable to assess the intracellular biological function of TRK receptors other than TRKB and the effect of ONO-7579 on normal cells at the concentration used in our experiment. In addition, only two GBC cell lines were used, and only in vitro.

Figure 6 depicts a schematic of our present findings. In summary, ONO-7579 showed suppressive effects on proliferation, invasion, and expression of VEGFs, that are thought to be associated with a poor prognosis. ONO-7579 is a promising candidate compound for the treatment of $\mathrm{GBC}$, at least in patients harboring wild-type $K R A S$, and further study is desired.

\section{Funding}

This study was supported by the Japan Society for the Promotion of Science KAKENHI Grant Number 26462146 and 16K10603.

\section{References}

1 Donohue JH, Stewart AK and Menck HR: The National Cancer Data Base report on carcinoma of the gallbladder, 1989-1995. Cancer 83(12): 2618-2628, 1998.

2 Eckel F and Schmid RM: Chemotherapy in advanced biliary tract carcinoma: a pooled analysis of clinical trials. Br J Cancer 96(6): 896-902, 2007.

3 de Moraes JK, Wagner VP, Fonseca FP, Vargas PA, de Farias $\mathrm{CB}$, Roesler $\mathrm{R}$ and Martins MD: Uncovering the role of brainderived neurotrophic factor/tyrosine kinase receptor B signaling in head and neck malignancies. J Oral Pathol Med, 2017. doi: 10.1111/jop.12611. [Epub ahead of print]

4 Rolfo C, Ruiz R, Giovannetti E, Gil-Bazo I, Russo A, Passiglia F, Giallombardo M, Peeters M and Raez L: Entrectinib: a potent new TRK, ROS1, and ALK inhibitor. Expert Opin Investig Drugs 24(11): 1493-1500, 2015.

5 Au CW, Siu MK, Liao X, Wong ES, Ngan HY, Tam KF, Chan DC, Chan QK and Cheung AN: Tyrosine kinase B receptor and BDNF expression in ovarian cancers - Effect on cell migration, angio-genesis and clinical outcome. Cancer Lett 281(2): 151$161,2009$.

6 Sclabas GM, Fujioka S, Schmidt C, Li Z, Frederick WA, Yang W, Yokoi K, Evans DB, Abbruzzese JL, Hess KR, Zhang W, Fidler IJ and Chiao PJ: Overexpression of tropomysin-related kinase B in metastatic human pancreatic cancer cells. Clin Cancer Res 11(2 Pt 1): 440-449, 2005. 
7 Fujikawa H, Tanaka K, Toiyama Y, Saigusa S, Inoue Y, Uchida $\mathrm{K}$ and Kusunoki M: High TrkB expression levels are associated with poor prognosis and EMT induction in colorectal cancer cells. J Gastroenterol 47(7): 775-784, 2012.

8 Kawamoto M, Onishi H, Ozono K, Yamasaki A, Imaizumi A, Kamakura S, Nakano K, Oda Y, Sumimoto H and Nakamura M: Tropomyosin-related kinase B mediated signaling contributes to the induction of malignant phenotype of gallbladder cancer. Oncotarget 8(22): 36211-36224, 2017.

9 Doebele RC, Davis LE, Vaishnavi A, Le AT, Estrada-Bernal A, Keysar S, Jimeno A, Varella-Garcia M, Aisner DL, Li Y, Stephens PJ, Morosini D, Tuch BB, Fernandes M, Nanda N and Low JA: An Oncogenic NTRK fusion in a patient with softtissue sarcoma with response to the tropomyosin-related kinase inhibitor LOXO-101. Cancer Discov 5(10): 1049-1057, 2015.

10 Ricciuti B, Brambilla M, Metro G, Baglivo S, Matocci R, Pirro M and Chiari R: Targeting NTRK fusion in non-small cell lung cancer: rationale and clinical evidence. Med Oncol 34(6): 105, 2017.

11 Milione M, Ardini E, Christiansen J, Valtorta E, Veronese S, Bosotti R, Pellegrinelli A, Testi A, Pietrantonio F, Fuca G, Wei G, Murphy D, Siena S, Isacchi A and De Braud F: Identification and characterization of a novel SCYL3-NTRK1 rearrangement in a colorectal cancer patient. Oncotarget 8(33): 55353-55360, 2017.

12 Drilon A, Siena S, Ou SI, Patel M, Ahn MJ, Lee J, Bauer TM, Farago AF, Wheler JJ, Liu SV, Doebele R, Giannetta L, Cerea G, Marrapese G, Schirru M, Amatu A, Bencardino K, Palmeri L, Sartore-Bianchi A, Vanzulli A, Cresta S, Damian S, Duca M, Ardini E, Li G, Christiansen J, Kowalski K, Johnson AD, Patel R, Luo D, Chow-Maneval E, Hornby Z, Multani PS, Shaw AT and De Braud FG: Safety and Antitumor Activity of the Multitargeted Pan-TRK, ROS1, and ALK Inhibitor Entrectinib: Combined Results from Two Phase I Trials (ALKA-372-001 and STARTRK-1). Cancer Discov 7(4): 400-409, 2017.

13 Horiuchi H, Kawamata H, Fujimori T and Kuroda Y: A MEK inhibitor (U0126) prolongs survival in nude mice bearing human gallbladder cancer cells with K-ras mutation: analysis in a novel orthotopic inoculation model. Int J Oncol 23(4): 957-963, 2003.

14 Sekine S, Shimada Y, Nagata T, Moriyama M, Omura T, Yoshioka I, Hori R, Matsui K, Sawada S, Okumura T, Yoshida $\mathrm{T}$ and Tsukada K: Establishment and characterization of a new human gallbladder carcinoma cell line. Anticancer Res 32(8): 3211-3218, 2012.

15 Matsushita S, Onishi H, Nakano K, Nagamatsu I, Imaizumi A, Hattori M, Oda Y, Tanaka M and Katano M: Hedgehog signaling pathway is a potential therapeutic target for gallbladder cancer. Cancer Sci 105(3): 272-280, 2014.

16 Hua Z, Gu X, Dong Y, Tan F, Liu Z, Thiele CJ and Li Z: PI3K and MAPK pathways mediate the BDNF/TrkB-increased metastasis in neuroblastoma. Tumour Biol, 2016. [Epub ahead of print]

17 Nakamura K, Martin KC, Jackson JK, Beppu K, Woo CW and Thiele CJ: Brain-derived neurotrophic factor activation of TrkB induces vascular endothelial growth factor expression via hypoxia-inducible factor-1alpha in neuroblastoma cells. Cancer Res 66(8): 4249-4255, 2006.

$18 \mathrm{Li} \mathrm{Z}$, Zhang Y, Tong Y, Tong J and Thiele CJ: Trk inhibitor attenuates the BDNF/TrkB-induced protection of neuroblastoma cells from etoposide in vitro and in vivo. Cancer Biol Ther 16(3): 477-483, 2015.

19 Chen B, Liang Y, He Z, An Y, Zhao W and Wu J: Autocrine activity of BDNF induced by the STAT3 signaling pathway causes prolonged TrkB activation and promotes human nonsmall-cell lung cancer proliferation. Sci Rep 6: 30404, 2016.

20 Esposito CL, D'Alessio A, de Franciscis V and Cerchia L: A cross-talk between TrkB and Ret tyrosine kinases receptors mediates neuroblastoma cells differentiation. PLoS One 3(2): e1643, 2008.

21 Nakamura Y, Suganami A, Fukuda M, Hasan MK, Yokochi T, Takatori A, Satoh S, Hoshino T, Tamura Y and Nakagawara A: Identification of novel candidate compounds targeting TrkB to induce apoptosis in neuroblastoma. Cancer Med 3(1): 25-35, 2014.

22 Odate S, Nakamura K, Onishi H, Kojima M, Uchiyama A, Nakano K, Kato M, Tanaka M and Katano M: TrkB/BDNF signaling pathway is a potential therapeutic target for pulmonary large cell neuroendocrine carcinoma. Lung Cancer 79(3): 205214, 2013.

23 Ozono K, Ohishi Y, Onishi H, Nakamura K, Motoshita J, Kato M, Nakanishi R, Nakamura $\mathrm{M}$ and Oda Y: Brain-derived neurotrophic factor/tropomyosin-related kinase B signaling pathway contributes to the aggressive behavior of lung squamous cell carcinoma. Lab Invest 97(11): 1332-1342, 2017.

24 Strock CJ, Park JI, Rosen M, Dionne C, Ruggeri B, Jones-Bolin S, Denmeade SR, Ball DW and Nelkin BD: CEP-701 and CEP751 inhibit constitutively activated RET tyrosine kinase activity and block medullary thyroid carcinoma cell growth. Cancer Res 63(17): 5559-5563, 2003.

25 Shabbir M and Stuart R: Lestaurtinib, a multitargeted tyrosine kinase inhibitor: from bench to bedside. Expert Opin Investig Drugs 19: 427-436, 2010.

26 Drilon A, Nagasubramanian R, Blake JF, Ku N, Tuch BB, Ebata K, Smith S, Lauriault V, Kolakowski GR, Brandhuber BJ, Larsen PD, Bouhana KS, Winski SL, Hamor R, Wu WI, Parker A, Morales TH, Sullivan FX, DeWolf WE, Wollenberg LA, Gordon PR, Douglas-Lindsay DN, Scaltriti M, Benayed R, Raj S, Hanusch B, Schram AM, Jonsson P, Berger MF, Hechtman JF, Taylor BS, Andrews S, Rothenberg SM and Hyman DM: A next-generation TRK kinase inhibitor overcomes acquired resistance to prior TRK kinase inhibition in patients with TRK fusion-positive solid tumors. Cancer Discov 7(9): 963-972, 2017.

27 Mohri D, Ijichi H, Miyabayashi K, Takahashi R, Kudo Y, Sasaki T, Asaoka Y, Tanaka Y, Ikenoue T, Tateishi K, Tada M, Isayama $\mathrm{H}$ and Koike K: A potent therapeutics for gallbladder cancer by combinatorial inhibition of the MAPK and mTOR signaling networks. J Gastroenterol 51(7): 711-721, 2016.

28 Kanthan R, Senger JL, Ahmed S and Kanthan SC: Gallbladder Cancer in the 21st Century. J Oncol 2015: 967472, 2015.

29 Mirzoeva OK, Das D, Heiser LM, Bhattacharya S, Siwak D, Gendelman R, Bayani N, Wang NJ, Neve RM, Guan Y, Hu Z, Knight Z, Feiler HS, Gascard P, Parvin B, Spellman PT, Shokat KM, Wyrobek AJ, Bissell MJ, McCormick F, Kuo WL, Mills GB, Gray JW and Korn WM: Basal subtype and MAPK/ERK kinase (MEK)-phosphoinositide 3-kinase feedback signaling determine susceptibility of breast cancer cells to MEK inhibition. Cancer Res 69(2): 565-572, 2009. 